\title{
CAPACIDADE FUNCIONAL DE INDIVÍDUOS COM LOMBALGIA EM UMA UNIDADE BÁSICA DE SAÚDE DE GETÚLIO VARGAS
}

\author{
Functional capacity of patients with low back pain of a healthcare center \\ from Getúlio Vargas city
}

\author{
Michele Dassi ${ }^{1}$; Arthiese Korb ${ }^{2}$
}

\begin{abstract}
${ }^{1}$ Fisioterapeuta. Graduada pela Universidade de Passo-Fundo - UPF. Pós-graduada em Fisioterapia Hospitalar pela Universidade de Passo Fundo. Especialista em Fisioterapia Ortopédica e Traumatológica URI/IOT

${ }^{2}$ Fisioterapeuta Graduada pela Universidade Federal de Santa Maria- UFSM. Doutora em Medicina: Ciências Médicas, pela Universidade Federal do Rio Grande do Sul- UFRGS. Docente do Programa de Pós-Graduação em Fisioterapia Ortopédica e Traumatológica URI/IOT.E-mail: arthi.korb@gmail.com
\end{abstract}

Data do recebimento: 09/10/2020 - Data do aceite: 17/03/2021

RESUMO: A dor lombar ou lombalgia é uma queixa muito comum, e afeta com maior frequência a população em seu período de vida mais produtivo. Há evidências de que os fatores psicossociais podem ser mais importantes do que os aspectos fisiológicos no desenvolvimento da dor crônica e da incapacidade. O presente estudo analisou os padrões de uma população atingida pela lombalgia e o quanto isso afeta seu dia a dia. Pesquisa caracterizada como um estudo descritivo, de cunho quantitativo. Composto por cinquenta voluntários com queixa de dor lombar, usuários da Unidade Básica de Saúde Santo-André, da cidade de Getúlio-Vargas/RS. Para coleta de dados foi aplicado um questionário sociodemográfico, Escala Visual Analógica de dor EVA e questionário de Roland e Morris. Dos participantes que compuseram a amostra, $80 \%$ são do sexo feminino, $60 \%$ sedentários. A grande maioria dos participantes relataram algum tipo de limitação funcional (78\%), no entanto, na mensuração da incapacidade funcional, a partir do questionário RolandMorris, 46\% dos indivíduos apresentaram incapacidade funcional e grande parte dos indivíduos deste estudo apresentaram dor de intensidade moderada. Observamos correlação entre maior incapacidade funcional e sintomas álgicos. Conclui-se que $46 \%$ dos indivíduos apresentaram perda da capacidade funcional devido a lombalgia.

Palavras-chave: Dor lombar. Coluna vertebral. Limitação da mobilidade. 
ABSTRACT: Low back pain is a common injury and it often affects the population in their most productive life span. There is evidence that psychosocial factors may be more important than physiological aspects in the development of chronic pain and disability. This study analyzed the patterns of a population affected by low back pain and how much it affects their daily lives. This is a descriptive quantitative study. The sample consisted of fifty users of Santo André Healthcare Center from Getulio Vargas city, all reporting low back pain. A socio-demographic questionnaire, Visual Analogue Pain Scale VAS and a Roland and Morris questionnaire were applied in order to collect data. $80 \%$ of the participants are female, among them $60 \%$ are sedentary. Most participants reported some type of functional limitation (78\%), however, in the measurement of functional disability, using the Roland-Morris questionnaire, $46 \%$ of the individuals had functional disability and moderate pain. It was observed a correlation between functional disability and pain symptoms. It is concluded that $46 \%$ of the individuals presented loss of functional capacity due to low back pain.

Keywords: Low back pain. Functional disability. Spine.

\section{Introdução}

A dor lombar ou lombalgia é uma queixa muito comum, e afeta com maior frequência a população em seu período de vida mais produtivo. A lombociatalgia apresenta uma grande incidência, causando diversos prejuízos aos seus portadores, sendo grande responsável pelas queixas de dor e levando a dificuldade nas atividades de vida diária (AVD'S), afastamento do trabalho e prejuízos sociais gerando impactos biopsicossociais e financeiros (LUO et al., 2004)

A dor lombar não decorre de doenças específicas, mas sim de um conjunto de causas, como por exemplo fatores sociodemográficos (idade, sexo, renda e escolaridade), comportamentais (fumo e baixa atividade física), exposições ocorridas nas atividades cotidianas (trabalho físico pesado, vibração, posição viciosa, movimentos repetitivos) e outros (obesidade, morbidades psicológicas) (MARRAS, 2000). Além disso, os fatores psicossociais aparecem como fundamentais no desenvolvimento da dor crônica e da incapacidade ((WOBY SR, URMSTON M, [s.d.])WOBY et al., 2007). A incapacidade em pacientes com dor lombar crônica varia de $11 \%$ a $76 \%$ (CÔTÉ et al., 2008).

Segundo Silva e colaboradores (2019)( SILVA et al., 2019), a incapacidade funcional conduz os pacientes a prejuízos socioeconômicos devido ao maior isolamento social, tendo, também, consequências psicológicas. Além disso, a incapacidade funcional associada à lombalgia exerce grande efeito negativo no bem-estar individual, gerando mais necessidade de apoio formal, informal e cuidados por longos períodos gerando altos custos para saúde pública e afetando, significativamente, a qualidade de vida dos indivíduos, levando ao afastamento do trabalho e aposentadoria por invalidez (RIBEIRO et al., 2018; SILVA; INUMARU, 2015).

A aplicação dos questionários é uma tentativa de quantificar a saúde, o impacto da 
patologia sobre as atividades diárias (NGUYEN THI PL; BRIANÇON S; EMPEREUR F, [s.d.]). Sabe-se que a utilização de questionários na pesquisa cientifica apresenta vantagens como menores gastos, anonimato, permite respostas convenientes ao momento, diminui as chances de influência do pesquisador nas respostas do entrevistado.

O presente estudo busca entender os padrões de uma população atingida pela lombalgia e o quanto isso afeta seu dia a dia, levando em consideração as características sociodemográficas e a capacidade funcional dos indivíduos.

A pesquisa é caracterizada como um estudo descritivo, de cunho quantitativo, de campo transversal. Composta por cinquenta voluntários com queixa de dor lombar, usuários da Unidade Básica de Saúde Santo -André, da cidade de Getúlio-Vargas/RS, no período de julho a novembro de 2019.

O projeto de pesquisa foi submetido ao Comitê de Ética em Pesquisa da Universidade Regional Integrada do Alto Uruguai e das Missões (URI), Câmpus de Erechim, tendo sido registrado com o número de protocolo CAAE $n^{\circ}$ 14021119.3.0000.5351. Obteve aprovação em 04 de julho de 2019, e autorizado pela Secretaria Municipal de Saúde do município de Getúlio Vargas.

Foram convidados a participar da pesquisa, indivíduos da unidade de saúde no momento que chegavam ou aguardavam atendimento e que se enquadraram nos critérios de inclusão, com idade superior a 18 anos de idade, de ambos os sexos com queixa de dor lombar. Para participar desse estudo os participantes assinaram um Termo de Consentimento Livre e Esclarecido.

Para coleta de dados foi aplicado um questionário sociodemográfico com dados sobre identificação, IMC, idade, escolaridade, profissão, tempo de trabalho, doenças associadas, medicamentos, atividade física, limitação diária e tratamento realizado; informações sobre a Escala Visual Analógica de dor EVA e questionário de Roland e Morris sobre incapacidade.

A EVA foi utilizada para quantificar a intensidade da dor, sendo graduada de acordo com pontuações ordinais: nenhuma dor (0), dor leve (1 a 2), moderada (3 a 5) e intensa (6 a 10) (TONIOLLI; PAGLIUCA, 2003).

O questionário Roland-Morris Brasil, específico para medir a incapacidade funcional de pacientes com lombalgia, é composto de 24 questões relacionadas às atividades de vida diária, dor e função. Para cada questão afirmativa é atribuído 1 ponto. O escore é a somatória dos valores, podendo-se obter uma pontuação mínima de " 0 " e uma pontuação máxima de "24". Este questionário tem como ponto de corte o escore "14", ou seja, os indivíduos avaliados com um escore igual ou maior que "14" são classificados como incapacitados funcionalmente (LOPES; CICONELLI; REIS, 2007).

A análise dos dados foi realizada por meio de estatística descritiva, com cálculo de porcentagem, média, desvio padrão, tabelas, tabelas de distribuição de frequência. Para correlação entre os resultados da percepção da dor com o questionário EVA e questionário Rolland-Morris foi utilizada a correlação não paramétrica rô de Spearman e apresentação de digrama de dispersão. Além disso, foi utilizado o coeficiente de correlação de Pearson para a relação entre tempo de sintomas de lombalgia e tempo de trabalho. As análises foram feitas utilizando-se a planilha Microsoft Excel e o software STATISTICA 5.0.

\section{Resultados}

Compuseram a amostra 50 pessoas, sendo $40(80 \%)$ do sexo feminino e $10(20 \%)$ do sexo masculino. A Tabela I mostra a média 
de idade (56,4 DP 16,00) e Média do IMC (27,0 DP 4,1) da amostra pesquisada.

A Tabela II apresenta a distribuição dos participantes por IMC. Observa-se que 16 indivíduos (32\%) apresentam peso normal para faixa etária e $68 \%$ dos participantes estão acima do peso, sendo 25 pré-obesos $(50 \%)$, 8 indivíduos em obesidade Grau I (16\%) e 1 indivíduo em obesidade Grau II (2\%).

Verificou-se que $20(40 \%)$ pessoas realizam atividades físicas e 30 (60\%) são sedentárias. A Tabela III apresenta os exercícios físicos realizados pelos participantes da pesquisa. Verifica-se que $50 \%$ dos participantes realizam caminhada, seguido pela hidroginástica, musculação e Pilates, com 10\%.
A análise mostrou que $11(22 \%)$ pessoas não relataram limitação na vida diária motivada pela lombalgia e 39 (78\%) apresentaram alguma limitação. A principal limitação está relacionada com os serviços do lar -20 $(40 \%)$. As outras 30(60\%) pessoas relataram diversas limitações, como caminhar, levantar e carregar peso, lascar lenha, ficar em pé, carpir e associações entre mais de uma atividade limitante.

A Tabela IV mostra a relação da amostra pesquisada entre sexo e escolaridade. Podese observar que as pessoas com escolaridade em nível de Educação Básica, completa ou incompleta soma $72 \%$ da amostra, sendo que $42 \%$ das pessoas têm Ensino Fundamental Incompleto.

Tabela I - Média de idade/IMC

\begin{tabular}{|c|c|c|c|c|c|c|c|c|c|}
\hline \multirow{2}{*}{ Sexo } & \multirow{2}{*}{$\mathbf{N}$} & \multicolumn{3}{|c|}{ Idade(anos) } & \multicolumn{5}{|c|}{ IMC $\left(\mathrm{kg} / \mathrm{m}^{2}\right)$} \\
\hline & & Mínimo & Máximo & Média & DP & Mínimo & Máximo & Média & DP \\
\hline Masculino & 10 & 27,0 & 78,0 & 54,5 & 19,0 & 25,9 & 34,3 & 29,3 & 3,0 \\
\hline Feminino & 40 & 20,0 & 79,0 & 53,1 & 15,5 & 19,1 & 35,6 & 26,4 & 4,2 \\
\hline Média & 50 & - & - & 56,4 & 16,0 & - & - & 27,0 & 4,1 \\
\hline
\end{tabular}

Tabela II - Índice de massa corpórea

\begin{tabular}{lcc}
\hline $\begin{array}{l}\text { Índice de Massa Corpórea } \\
\text { (IMC) }\end{array}$ & N & \% \\
\hline Normal (18,5 - 24,9) & 16 & 32,0 \\
Pré-obeso (25,0 - 29,9) & 25 & 50,0 \\
Obesidade Classe I (30 - 34,9) & 8 & 16,0 \\
Obesidade Classe II (35,0 - & & \\
39,9) & 1 & 2,0 \\
\hline Total & 50 & 100,0 \\
\hline
\end{tabular}

Tabela III - Exercício Físico

\begin{tabular}{lccc}
\hline \multirow{2}{*}{ Atividade Física } & n & \multicolumn{2}{c}{ Proporção (\%) } \\
\cline { 3 - 4 } & & n total & n ativos \\
\hline Não praticam & 30 & 60,0 & - \\
Caminhada & 10 & 20,0 & 50,0 \\
Dança & 1 & 2,0 & 5,0 \\
Hidroterapia & 2 & 4,0 & 10,0 \\
Hidroterapia, & 1 & 2,0 & 5,0 \\
atividades em grupo & & & \\
Musculação & 2 & 4,0 & 10,0 \\
Musculação, dança & 1 & 2,0 & 5,0 \\
Musculação, futsal & 1 & 2,0 & 5,0 \\
Pilates & 2 & 4,0 & 10,0 \\
\hline Total & 50 & $\mathbf{1 0 0 , 0}$ & $\mathbf{1 0 0 , 0}$ \\
\hline
\end{tabular}


A tabela $\mathrm{V}$ apresenta as estatísticas das variáveis tempo de trabalho e tempo de sintomas de lombalgia. Os desvios padrões elevados mostram uma variabilidade muito grande no tempo de trabalho dos participantes da pesquisa e, consequentemente, no tempo de sintomas. A Tabela VI mostra, conside- rando a distribuição das médias de tempo de lombalgia e tempo de trabalho, que, pelo coeficiente de Correlação de Pearson, $r=$ 0,89 , existe uma forte correlação entre ambas, indicando que quanto maior for o tempo de trabalho das pessoas, maior será o tempo de sintomas de lombalgia.

Tabela IV - Distribuição da amostra por sexo e escolaridade

\begin{tabular}{lccc}
\hline \multicolumn{1}{c}{ Escolaridade } & \multicolumn{2}{c}{ Sexo } & Total \\
\cline { 2 - 3 } & Masculino & Feminino & $6,00 \%$ \\
\hline Ensino Fundamental & $2,00 \%$ & $4,00 \%$ & $42,00 \%$ \\
Ensino Fundamental Incompleto & $8,00 \%$ & $34,00 \%$ & $24,00 \%$ \\
Ensino Médio & $2,00 \%$ & $22,00 \%$ & $2,00 \%$ \\
Ensino Médio incompleto & - & $2,00 \%$ & $16,00 \%$ \\
Ensino Superior & $2,00 \%$ & $14,00 \%$ & $4,00 \%$ \\
Ensino Superior incompleto & $4,00 \%$ & - & $2,00 \%$ \\
Ensino Técnico & - & $2,00 \%$ & $4,00 \%$ \\
Pós-graduação & $2,00 \%$ & $2,00 \%$ & $\mathbf{1 0 0 , 0 0 \%}$ \\
\hline Total & $\mathbf{2 0 , 0 0 \%}$ & $\mathbf{8 0 , 0 0 \%}$ & \\
\hline
\end{tabular}

Tabela V - Estatísticas do tempo de sintomas de lombalgia e tempo de trabalho

\begin{tabular}{lccccc}
\hline \multicolumn{1}{c}{ Variável (anos) } & N & Mínimo & Máximo & Média & Desvio Padrão \\
\hline Tempo de trabalho* & 49 & 0,13 & 74,00 & 26,83 & 22,98 \\
Tempo de sintomas & 50 & 0,08 & 50,0 & 10,50 & 11,12 \\
\hline
\end{tabular}

* Um participante não relatou tempo de trabalho

Tabela VI - Distribuição de frequências da duração dos sintomas de lombalgia e tempo de trabalho

\begin{tabular}{lcccccc}
\hline \multirow{2}{*}{ Tempo (anos) } & & \multicolumn{3}{c}{ Duração da dor (anos) } & \multicolumn{2}{c}{ Tempo de trabalho(anos) } \\
\cline { 2 - 7 } & Fi* $^{*}$ & $\mathbf{\%}$ & Média $^{* *}$ & DP & Média** $^{* *}$ & DP \\
\hline 0 a 1 & 9 & 18.4 & 0.5 & 0.3 & 16.2 & 19,0 \\
$\mathbf{2}$ a 5 & 15 & 30.6 & 3.8 & 1.1 & 22.5 & 20.7 \\
$\mathbf{6}$ a 10 & 10 & 20.4 & 9.6 & 1.3 & 23.5 & 18.5 \\
$\mathbf{1 1}$ a 15 & 5 & 10.2 & 14.4 & 1.3 & 40,0 & 26.3 \\
$\mathbf{1 6}$ a 20 & 5 & 10.2 & 20,0 & 0,0 & 26.4 & 30.1 \\
Mais de 20 & 5 & 10.2 & 37,0 & 8.4 & 52.6 & 18.4 \\
Total & 49 & 100,0 & 14,2 & - & 30,2 & - \\
\hline
\end{tabular}

* Como um dos participantes não declarou o tempo de serviço, os cálculos foram feitos para 49.

** Correlação de Pearson. $\mathrm{R}=0,89 . \mathrm{p}=0,004$. 
A tabela VII mostra que, mesmo com lombalgia, $15(30 \%)$ das pessoas não utilizam nenhuma forma de tratamento. Das pessoas que buscam tratamento, foi utilizado medicamento associado ao tratamento fisioterápico (17 pessoas $-34,7 \%$ ).

Tabela VII - Tratamento

\begin{tabular}{lcc}
\hline Tratamento & $\mathbf{n}$ & $\mathbf{\%}$ \\
\hline Sem tratamento & 15 & 30,0 \\
Procedimento cirúrgico & 1 & 2,0 \\
Fisioterapia & 3 & 6,1 \\
Fisioterapia e massagem & 2 & 4,1 \\
Medicamentos & 4 & 8,2 \\
Medicamentos e Fisioterapia & 17 & 34,7 \\
Medicamentos, Fisioterapia & 3 & 6,1 \\
e massagem & & 8,2 \\
Medicamentos e massagens & 4 & 2,0 \\
Medicamentos e Pilates & 1 & $\mathbf{1 0 0 , 0}$ \\
\hline Total & $\mathbf{5 0}$ & \\
\hline
\end{tabular}

A maioria das pessoas não apresentam doenças associadas a lombalgia (29-58\%), como pode ser visto na Tabela VIII.

A maioria dos participantes apresentou dor moderada, 70\% segundo a Escala Visual Analógica de dor (EVA), seguindo de 26\% com dor intensa e $4 \%$ com dor leve como podemos ver na Tabela IX.
Tabela VIII - Doenças associadas

\begin{tabular}{lcc}
\hline Doença Associada & $\mathbf{n}$ & $\mathbf{\%}$ \\
\hline Não apresentam & 29 & 58,0 \\
Colesterol alto & 3 & 6,0 \\
Hipertensão & 3 & 6,0 \\
Parkinson & 3 & 6,0 \\
Fibromialgia & 2 & 4,0 \\
Hipotireoidismo & 2 & 4,0 \\
Arritmia cardíaca & 1 & 2,0 \\
Artrose & 1 & 2,0 \\
Bursite trocantérica & 1 & 2,0 \\
Colesterol alto, & 1 & 2,0 \\
hipertireoidismo & 1 & 2,0 \\
Hipertensão & 1 & 2,0 \\
Hipertensão, & & 2,0 \\
hipertireoidismo & 1 & $\mathbf{1 0 0 , 0}$ \\
Hipertireoidismo, bursite & & \\
em ombro, cervicalgia & & \\
Tendinite em ombro & & \\
\hline Total & & \\
\hline
\end{tabular}

Na mensuração da incapacidade funcional, a partir do questionário Roland-Morris, tabela $\mathrm{X}$, foi observado que $46 \%$ dos indivíduos foram classificados como portadores de incapacidade funcional devido à lombalgia.

Tabela IX - Caracterização da dor de acordo com EVA

\begin{tabular}{lccccccc}
\hline \multicolumn{1}{c}{ EVA } & $\mathbf{N}$ & $\mathbf{\%}$ & Mínimo & Máximo & Mediana & Média & DP* \\
\hline Leve (1 a 2) & 2 & 4,0 & 1,0 & 2,0 & 1,5 & 2,0 & 0,7 \\
Moderada (3 a 7) & 35 & 70,0 & 3,0 & 7,0 & 5,0 & 5,4 & 1,1 \\
Intensa (8 a 10) & 13 & 26,0 & 8,0 & 10,0 & 9,0 & 9,0 & 1,0 \\
\hline Total & 50 & 100,0 & - & - & - & - & - \\
\hline
\end{tabular}

* DP: desvio padrão 
Tabela $\mathbf{X}$ - Nível de incapacidade funcional questionário Rolland-Morris

\begin{tabular}{lcc}
\hline Pontuação & $\mathbf{n}$ & $\mathbf{\%}$ \\
\hline De 0 a 6 pontos & 12 & 24,0 \\
De 7 a 13 pontos & 15 & 30,0 \\
14 ou mais pontos & & \\
(incapacidade) & 23 & 46,0 \\
\hline Total & $\mathbf{5 0}$ & $\mathbf{1 0 0}$ \\
\hline
\end{tabular}

* De acordo com Lopes AD, Ciconelli RM, Reis FB.

Considerando que os resultados dos instrumentos EVAe Rolland-Morris são escores, foi calculada a correlação entre os resultados da percepção da dor pelo questionário EVA e pelo questionário Rolland-Morris, para incapacidade funcional, utilizando-se a correlação não paramétrica rô de Spearman, obtendo-se $r=0,588$ ( $<<0,01)$, o que mostra regular paridade nos resultados detectados pelas duas escalas, considerando, principalmente, o grau de subjetividade do instrumento EVA. O diagrama de dispersão da Figura 01 mostra a relação positiva entre os resultados dos dois instrumentos, ou seja, é possível observar que os valores mais altos do Questionário de Rolland-Morris (maior incapacidade) correspondem aos valores mais altos da escala EVA (maior dor). A dispersão dos pontos mostra indícios da paridade dos resultados. Como alegado anteriormente, a subjetividade da mensuração da dor pode ser um ingrediente dificultador na obtenção de melhor correlação entre os resultados das duas escalas.

\section{Discussão}

A dor lombar aparece como um grande fator incapacitante, afetando a população e levando a limitações ou incapacidades funcionais, dificultando o desenvolvimento das atividades de vida diária, além de restrição na participação dos indivíduos na sociedade e reduzindo os padrões esperados de qualidade de vida (SAMPAIO et al., 2005).

Neste contexto, alguns estudos como o de (CARAVIELLO et al., 2005; MASCARENHAS; SANTOS, 2011; ROMERO et al., 2018), demonstraram que grande parte

Figura 01 - Diagrama de dispersão entre os resultados dos instrumentos EVA e Rolland-Morris

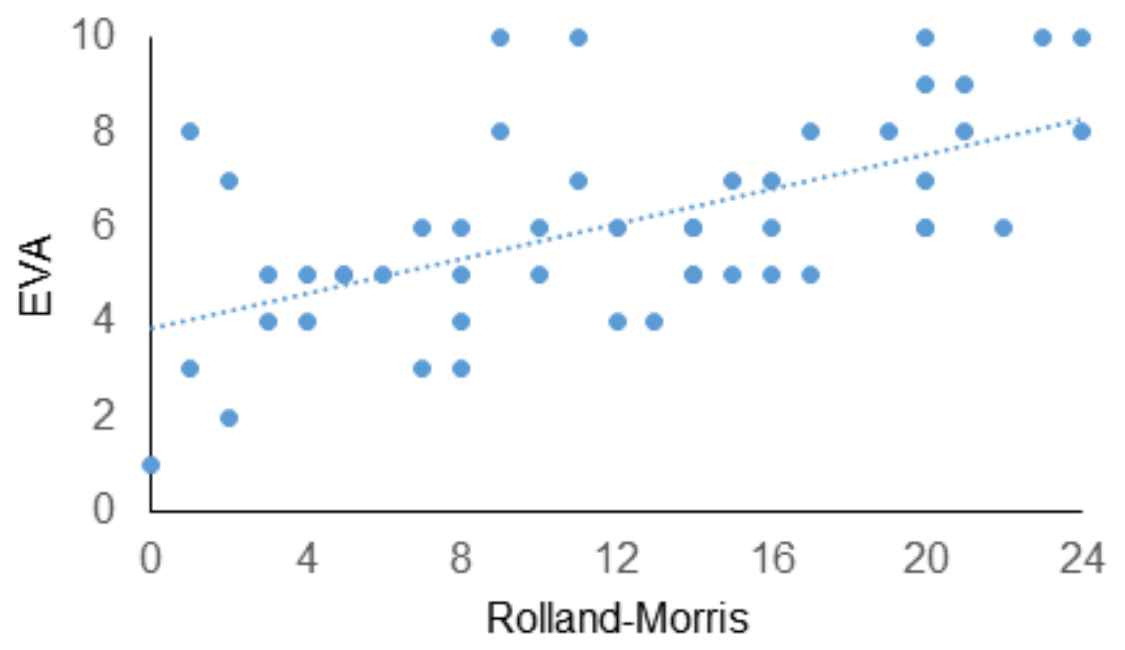


da população com queixa de dor lombar são mulheres. Esses dados corroboram com nossos achados, onde $80 \%$ da população estudada era composta por mulheres. As mulheres, cada vez mais, combinam a realização de tarefas domésticas com o trabalho fora de casa, onde estão expostas a cargas ergonômicas, principalmente repetitividade, posição viciosa e trabalho em grande velocidade. Além disso, o sexo feminino apresenta algumas características anátomo-funcionais (menor estatura, menor massa muscular, menor massa óssea, articulações mais frágeis e menos adaptadas ao esforço físico pesado, maior peso de gordura) que podem colaborar para o surgimento das dores lombares crônicas (ROMERO et al., 2018).

Em relação à idade, observou-se a média de 56,4 anos, a maioria dos individuos iniciaram suas atividades laborais na agricultura ainda na infância e alguns mantiveram suas atividades com o passar dos anos. Além disso, o fator idade pode ser justificado pelo fato de que a lombalgia pode acometer, principalmente, indivíduos economicamente ativos, os quais podem estar expostos a cargas excessivas de trabalho (MASCARENHAS; SANTOS, 2011).

Em relação ao tempo de trabalho e aos sintomas álgicos, os resultados demonstraram que, quanto maior for o tempo de trabalho maior será o tempo de lombalgia. O tempo de sintomas apresentou uma média de 10,5 anos, com média de 26,83 anos de trabalho, semelhante ao demonstrado no estudo de (TSUKIMOTO et al., 2006), onde o tempo médio da sintomatologia foi de 8,4 anos, considerando, portanto, em torno de uma década de sintomas álgicos. Destacamos a relevância do tempo de duração da dor lombar: independente da especificidade da dor contínua e de quanto mais longo sua perduração, por ventura, afeta muitos aspectos da vida, podendo levar a distúrbios do sono, depressão e irritabilidade (MASCA-
RENHAS; SANTOS, 2011). Diante desses sintomas podemos sugerir, além do tempo laboral, a influência sobre os hábitos de vida dessa populacao: sobrepeso, sedentarismo, levando à diminuição da funcionalidade do indivíduo e dores intensas.

Os resultados monstraram que a maioria dos indivíduos apresentaram o IMC fora da normalidade para sua faixa etária, com $68 \%$ dos índividuos acima do peso normal. Silva (SILVA; FASSA; VALLE, 2004) relata que quando há uma carga extra, cuja estrutura ósteo-músculo-articular é obrigada a sustentar, esta pode alterar o equilíbrio biomecânico do corpo e, consequentemente, aumentar o risco de dor lombar em pessoas com sobrepeso e obesidade. Neste contexto, nossos achados mostram que $60 \%$ do público estudado eram sedentários. Observa-se resultado parecido com o estudo de Bento (PAIVA; SIQUEIRA, 2009), onde somente $43,3 \%$ dos indivíduos realizavam algum tipo de atividade física. Esse resultado pode reforçar evidências de que indivíduos com dor, relatam uma intolerância às atividades físicas, sendo que nesses casos, o medo da dor é mais provocativo que o movimento realizado, o que predispõe ao sedentarismo, à instalação da incapacidade e com isso a piora da dor. O nível educacional observado, teve uma prevalência de ensino básico completo e incompleto. Sabe-se que o nível da escolaridade afeta a capacidade global dos indivíduos de enfrentar os desafios do cotidiano (GOMES et al., 2015).

A grande maioria dos participantes relataram alguma limitação funcional (78\%). A principal limitação estava relacionada com os serviços do lar. De acordo com Ocarino (OCARINO et al., 2009), a lombalgia pode levar a uma deficiência tanto no desempenho funcional quanto na capacidade física. Nesse aspecto podemos sugerir uma relação ao sobrepeso e sedentarismo observados. Além disso, 46\% dos indivíduos foram classificados como portadores de incapacidade 
funcional devido à lombalgia, segundo o questionário de Roland e Morris, mostrando resultados contrários aos de outros estudos, como no estudo de Ocarino (OCARINO et al., 2009), em que apenas 13,3\% dos indivíduos da amostra apresentaram incapacidade segundo o questionário de Rolland-Morris. Já, Mascarenhas e Santos (2011), observaram que $23,5 \%$ dos indivíduos apresentaram incapacidade funcional. Segundo estudo de Bento (PAIVA; SIQUEIRA, 2009) a dor lombar crônica não específica, raramente incapacita totalmente uma pessoa para exercer as atividades do cotidiano, entretanto, pode limitar, parcial e temporariamente e, muitas vezes, de forma recorrente. Esses resultados contrários podem ser justificados devido à forte intensidade de dor dos indivíduos, levando a limitações na funcionalidade e a falta de atividades físicas, gerando cada vez mais dor, além do aspecto psicossocial que apresenta grande influência na incapacidade em indivíduos com lombalgia (SALVETTI et al., 2012).

Além da incapacidade observada nos indivíduos, os resultados obtidos pela Escala Visual Analógica de dor (EVA) mostrou que $70 \%$ dos participantes apresentaram dor de intensidade moderada, resultado semelhante ao estudo de (MANN et al., 2009) onde 50\% dos indivíduos com dor lombar apresentavam dor intensa, segundo a EVA. Já, (MASCARENHAS; SANTOS, 2011) obtiveram relato de dor intensa em $70,6 \%$ dos indivíduos. (DE ABREU et al., 2008) em seu estudo encontrou uma forte correlação entre intensidade da dor com grau de incapacidade, sugerindo que a intensidade da dor pode ser um fator importante na manutenção e aumento da incapacidade, corroborando com nossos achados onde foi possível observar a correlação entre incapacidade funcional e intensidade de dor, ou seja quanto maior a incapacidade, maior a intensidade da dor observada.

\section{Considerações Finais}

Grande parte dos indivíduos deste estudo $(70 \%)$ apresentaram dor de intensidade moderada, segundo a escala EVA (78\%) relataram algum tipo de limitação funcional, entre elas as atividades como: serviços do lar $(40 \%)$, já $(60 \%)$, relataram limitações como, dificuldade de caminhar; levantar e carregar peso; além de apresentarem associações entre mais de uma atividade, as quais podem ser agravadas pela ausência de tratamento adequado, assim como, pelo sedentarismo e sobrepeso observados. Entretanto, na mensuração da incapacidade funcional, a partir do questionário Roland-Morris, somente 46\% dos indivíduos apresentaram incapacidade funcional, mas foi possível observar o aumento da incapacidade correlacionado com a maior intensidade da dor. Portanto, observa-se a presença de incapacidade associada a dor lombar.

Considerando a relevância desse assunto, ressalta-se a importância das informações sobre o problema e seu impacto na qualidade de vida, proporcionando conhecimentos fundamentais para a promoção e prevenção da saúde. Soma-se a isso, a necessidade da realização de novas pesquisas, a fim de permitir uma abordagem mais eficiente, com o objetivo de melhorar a assistência a essa população. Cabe destacar o papel do profissional fisioterapeuta junto a esses indivíduos, tanto para a prevenção como para tratamento e assim reduzir os riscos de incapacidade associados a essa disfunção. 


\section{REFERÊNCIAS}

CARAVIELLO, E. Z.; WASSERSTEIN, S.; CHAMLIAN, T. R.; MASIERO, D. Avaliação da dor e função de pacientes com lombalgia tratados com um programa de Escola de Coluna. Acta Fisiatrica, v. 12, n. 1, p. 11-14, 2005.

CÔTÉ, P.; BALDWIN, M. L.; JOHNSON, W. G.; FRANK, J. W.; BUTLE, R. J. Patterns of sickleave and health outcomes in injured workers with back pain. European Spine Journal, v. 17, n. 4, p. 484-493, 2008.

DE ABREU, A. M.; FARIA, C. D. C. M.; CARDOSO, S. M. V.; SALMELA, L. F. T. The Brazilian version of the fear avoidance beliefs questionnaire. Cadernos de Saude Publica, v. 24, n. 3, p. 615623, 2008.

GOMES, G. D. C.; TEIXEIRA-SALMELA, L. F.; FONSECA, B. E.; FREITAS, F. A. S.; FONSECA, M. L. M.; PACHECO, B. D.; GONÇALVES, M. R.; CARAMELLI, P. Age and education influence the performance of elderly women on the dual-task timed up and go test. Arquivos de Neuro-Psiquiatria, v. 73, n. 3, p. 187-193, 2015.

LUO, X.; PIETROBON, R.; SUN, S. X.; LIU, G. G.; HEY, L. Estimates and Patterns of Direct Health Care Expenditures Among Individuals With Back Pain in the United States. Spine, v. 29, n. 1, 2004.

MANN, L.; KLEINPAUL, J. F.; WEBER, P.; MOTA, C. B.; CARPES, F. P. Efeito do treinamento de Isostretching sobre a dor lombar crônica: um estudo de casos. Motriz, v. 15, n. 1, p. 50-60, 2009.

MARRAS, W. S. Occupational low back disorder causation and control. Ergonomics, v. 43, n. 7, p. 880-902, 2000.

MASCARENHAS, C. H. M.; SANTOS, L. S. Evaluation of pain and functional capacity in patients with chronic low back pain. J Health Sci Institute, v. 29, n. 3, p. 205-208, 2011.

NGUYEN THI PL, BRIANÇON S, EMPEREUR F, G. F. Factors Determining Inpatient Satisfaction With Care. Social Science \& Medicine, v.54, n.4, p. 493-504, 2002.

OCARINO, J. M.; GONÇALVES, G. G. P.; VAZ, D. V.; CABRAL, A. A. V.; PORTO, J. V..; SILVA, M. T. Correlação entre um questionário de desempenho funcional e testes de capacidade física em pacientes com lombalgia. Revista Brasileira de Fisioterapia, v. 13, n. 4, p. 343-349, 2009.

BENTO, A.A.C., PAIVA A.C.S., SIQUEIRA F.B Correlação entre incapacidade, dor - Roland Morris, e capacidade funcional - SF-36 em indivíduos com dor lombar crônica não específica Scientia, v.2, n.1, 2009.

RIBEIRO, R. P.; SEDREZ, J. A.; CANDOTTI, C. T .; VIEIRA, A. Relação entre a dor lombar crônica não específica com a incapacidade, a postura estática e a flexibilidade. Fisioterapia e Pesquisa, v. 25, n. 4, p. 425-431, 2018.

ROMERO, D. E.; SANTANA, D.; BORGES, P.; MARQUES, A.; CASTANHEIRA, D.;

RODRIGUES, J. M.; SABBADINI, L. Prevalência, fatores associados e limitações relacionados ao problema crônico de coluna entre adultos e idosos no Brasil. Cadernos de Saude Pública, v. 34, n. 2, p. e00012817, 2018.

SALVETTI, M. DE G.; PIMENTA, C. A. M.; BRAGA, P. E.; CORRÊA, C. F. Incapacidade 
relacionada à dor lombar crônica: prevalência e fatores associados Revista da Escola de Enfermagem da USP, v.46, n. spe, 2012.

SAMPAIO, R.; MANCINI, M. C.; GONÇALVES, G. G. P.; BITTENCOURT, N. F. N.; MIRANDA, A. D; FONSECA, S. T. Aplicação da classificação internacional de funcionalidade, incapacidade e saúde (CIF) na prática clínica do fisioterapeuta. Aplicação da classificação internacional de funcionalidade, incapacidade e saúde (CIF) na prática clínica do fisioterapeuta, v. 9, n. 2, p. 129-136, 2005.

SILVA, J. P. DA; JESUS-MORALEIDA, F.; FELÍCIO, D. C.; QUEIROZ, B. Z.; FERREIRA, M. L.; PEREIRA, L. S. M. Fatores biopsicossociais associados com a incapacidade em idosos com dor lombar aguda: estudo BACE-Brasil Ciência \& Saúde Coletiva, v. 24, n.7, 2019.

SILVA, M. C. DA; FASSA, A. G.; VALLE, N. C. J. Dor lombar crônica em uma população adulta do Sul do Brasil: prevalência e fatores associados Cadernos de Saúde Pública, v.20, n. 2, p. 377-385, 2004.

SILVA, P. H. B. DA; INUMARU, S. M. S. M. Dor Lombar Crônica- Correlação Entre Intensidade De Dor E Incapacidade : Dados Preliminares, v. 71, 2015.

TSUKIMOTO, G. R. Avaliação longitudinal da Escola de Postura para dor lombar crônica através da aplicação dos questionários Roland Morris e Short Form Health Survey (SF-36). Acta Fisiátrica, v. 13, n. 2, p. 63-69, 2006.

WOBY SR, URMSTON M, W. P. S. Mediates the reatin between pain-reated fear and outcome in chronic low back pain patients. European Journal Of Pain, v. 11, n. 7, p.711-8, 2007. 
